\title{
Effets de la Fertilisation à Base de la Biomasse du Sida cordifolia L. sur les Performances Agronomiques et la Rentabilité Économique de la Tomate (Lycopersicum esculentum Mill.) en Culture Irriguée
}

\author{
Souley Maman Sadi, Msc \\ Université Dan Dicko Dankoulodo de Maradi,Maradi, Niger \\ Département de gestion des ressources naturelles/ INRAN Niamey, Niger \\ Addam Kiari Saidou, \\ Département de gestion des ressources naturelles/ INRAN Niamey, Niger \\ Mourou Boube, \\ Université Dan Dicko Dankoulodo de Maradi, Maradi, Niger \\ Jens B. Aune, \\ Department of International Environment and Development Studies, \\ Norwegian University of Life Sciences, Norway
}

\section{Résumé}

L'utilisation démesurée des engrais minéraux et produits phytosanitaires en maraichage causent des sérieux problèmes sanitaires et environnementaux. Une étude a été conduite durant deux campagnes (2017 et 2018) à la station piscicole de Mollo située à environ $30 \mathrm{~km}$ au sud-est de Niamey sur l'incorporation de la biomasse du Sida cordifolia (BSC) comme fertilisant pour la culture de la tomate. L'objectif est d'évaluer les effets de la fertilisation à base de la BSC sur la production et la rentabilité de la tomate Roma VF en culture irriguée. Six traitements y compris le témoin : T0 $=0$ t. ha1 ; $\mathrm{T} 1=20 \mathrm{t}$. ha-1 de la fumure organique ; T2=20 t. ha-1; T3=30 t. ha-1 ; T4=40 t. ha- 1 et T5=50 t. ha- 1 de la BSC ont été répartis dans un dispositif en 4 blocs complètement randomisés. La BSC a été incorporée dans le sol 2 semaines avant le repiquage. Les éléments physico chimiques $(\mathrm{N}, \mathrm{P}, \mathrm{K}, \mathrm{pH}$, $\mathrm{C}, \mathrm{C} / \mathrm{N}) \mathrm{du}$ sol ont été déterminés avant et après les essais. Les données collectées ont été soumises à une ANOVA au seuil de 5\%. La rentabilité économique des traitements a été déterminée à travers le rapport valeur sur coût (RVC). Les résultats ont montré une amélioration de teneurs des éléments du sol après les deux campagnes. Les traitements ont influencé la hauteur de plants, la date de 50\% floraison ainsi que le rendement. En 2017, le rendement le plus élevé a été obtenu au niveau du traitement T4 (24,3 t. ha-1) et le témoin 
absolu (T0) le plus faible avec 10,02 t. ha-1. Pour la campagne 2018, les valeurs du rendement varient de 13,82 t. ha-1 (T0) à 33,68 t. ha-1 (T4). Des variabilités significatives de RVC entre les traitements et les années $(\mathrm{P}<0,05)$ ont été obtenues. Les résultats de deux campagnes montrent que le traitement T3 (30 t. ha-1 de la BSC) a toujours était le plus rentable que les autres traitements. Cette étude est d'autant plus intéressante que l'adoption de la fertilisation à base de la biomasse du Sida cordifolia pourrait permettre à la fois d'augmenter durablement la production de la tomate et la réduction de la prolifération du Sida cordifolia L. dans le terroir.

Mots clés : Fertilisation, performance Agronomique, Sida cordifolia L., Tomate, valorisation, irrigation

\title{
Effects of Fertilization Based on the Biomass of Sida cordifolia L. on the Agronomic Performance and Economic Profitability of Tomatoes (Lycopersicum esculentum Mill.) in Irrigated Crops
}

\author{
Souley Maman Sadi, Msc \\ Université Dan Dicko Dankoulodo de Maradi,Maradi, Niger \\ Département de gestion des ressources naturelles/ INRAN Niamey, Niger \\ Addam Kiari Saidou, \\ Département de gestion des ressources naturelles/ INRAN Niamey, Niger \\ Mourou Boube, \\ Université Dan Dicko Dankoulodo de Maradi, Maradi, Niger \\ Jens B. Aune, \\ Department of International Environment and Development Studies, \\ Norwegian University of Life Sciences, Norway
}

\begin{abstract}
The excessive use of mineral fertilizers and plant protection products in market gardening is causing serious health and environmental problems. A study was conducted during two campaigns (2017 and 2018) at the Molli Mollo fish farm located about $30 \mathrm{~km}$ southeast of Niamey on the incorporation of the biomass of Sida cordifolia (BSC) as a fertilizer for tomato cultivation. The objective is to assess the effects of BSC fertilization on the production and profitability of the Roma VF tomato in irrigated crops. Six treatments
\end{abstract}


including the control: $\mathrm{T} 0=0 \mathrm{t}$. ha- $1 ; \mathrm{T} 1=20 \mathrm{t}$. ha- 1 of organic manure; $\mathrm{T} 2=20$ t. ha-1; T3=30 t. ha-1; T4=40 t. ha-1 and T5=50 t. ha-1 of the BSC were distributed in a fully randomized 4-block system. The BSC was incorporated into the soil 2 weeks before the tomato was transplanted. The physicochemical elements $(\mathrm{N}, \mathrm{P}, \mathrm{K}, \mathrm{pH}, \mathrm{C}, \mathrm{C} / \mathrm{N})$ of the soil were determined before and after the tests. The data collected were submitted to an ANOVA at the 5\% threshold. The economic profitability of the treatments was determined through the value-to-cost ratio (VCR). The results showed an improvement in soil content after both campaigns. Treatments influenced plant height, $50 \%$ flowering date and yield. In 2017, the highest performance was achieved at the T4 treatment level (24.3 t. ha-1) and the lowest absolute control (T0) at 10.02 t. ha-1. For the 2018 campaign, yield values range from 13.82 t. ha-1 (T0) to 33.68 t. ha-1 (T4). Significant variabilities in VCR between treatments and years (P0.05) were obtained. The results of two campaigns show that the T3 treatment (30 t. ha-1 of the BSC) was always the most profitable than the other treatments. This study is all the more interesting because the adoption of fertilization based on the biomass of Sida cordifolia could allow both the sustainable increase of tomato production and the reduction of the proliferation of Sida cordifolia L. in the terroir.

Keywords : Fertilization, Agronomic performance, Sida cordifolia L. Tomato, Valorization, Irrigation

\section{Introduction}

Le maraichage est un secteur agricole vital en Afrique subsaharienne en raison de la valeur nutritive et des revenus économiques importants qu'il génère. En effet, ce secteur a la capacité de servir de moteur pour la diversification agricole et économique en orientant la production vers les marchés locaux ou d'exportation (Weinberger and Lumpkin, 2007).

$\mathrm{Au}$ Niger, se secteur connait une croissance exponentielle due au climat qui devient de plus en plus défavorable à la culture pluviale (Kiari, Aboubacar, et al., 2013). Les cultures maraichères constituent un véritable rempart aux cultures principales soit comme complément alimentaire soit comme source de revenu (Adam, 1995).

C'est le cas de la culture de tomate (Lycopersicum esculentum Mill.) qui constitue une activité lucrative pour de nombreux producteurs. Au Niger, la Tomate est la troisième culture maraichère après l'oignon et le chou. En 2018, elle est cultivée sur une superficie de 10507,55 ha avec un rendement moyen de 23,33 t. ha-1 (RECA, 2018). Ce rendement est très faible par rapport au potentiel de la tomate. Dans d'autres pays, des rendements d'environ 100 t. ha-1sont obtenus (Laterrot, 1994). 
Ce faible rendement, favorisé par un mauvais développement des plantes, est dû à la pression élevée des maladies, nématodes parasites des plantes et insectes nuisibles (Adamou, Garba, et al., 2017) et à la faible fertilité du sol. En effet, les besoins nutritionnels de la tomate sont énormes surtout en $\mathrm{N}$ et $\mathrm{K}$, or les engrais minéraux coûtent chers et ne sont pas à la portée de tous les paysans. Aussi leur utilisation abusive pollue les nappes phréatiques et provoque la salinisation des sols (Michel, Pierre, et al., 2016). Par conséquent ils compromettent la fertilité des sols et la qualité des fruits.

Il est donc nécessaire de faire recours à des fertilisants capables de fertiliser des sols tout en maintenant leur équilibre écologique. Les engrais organiques ont pour rôle d'améliorer la structure des sols, les enrichissent en éléments fertilisants et limitent la consommation de l'eau par les plantes. Malheureusement, les engrais organiques (fumure, bouse de vache), ne sont pas facilement disponibles. Mais d'autres sources de matières organiques plus accessibles et disponibles pour les agriculteurs existent. C'est le cas de Sida cordifolia L. qui est une plante herbacée de la famille des malvacées considérée comme une espèce envahissante et sans grande utilité au Niger. Ce travail a pour objectif d'évaluer les effets de la fertilisation à base de la BSC sur la production et la rentabilité de la tomate ROMA VF en culture irriguée.

\section{Matériels et méthode Site d'étude}

L'étude a été conduite au niveau de la station piscicole de Molli du Centre Régional de la Recherche Agronomique (CERRA) de Kollo situé aux coordonnées : N 13.29088 ; E 2.07535 à $30 \mathrm{~km}$ au Sud-Est de Niamey (figure $1)$.

Le climat de la zone d'étude est de type Sahélo-sahélien avec une pluviométrie annuelle moyenne de $482 \pm 65,80 \mathrm{~mm}$. Les températures moyennes gravitent autour de $30^{\circ} \mathrm{C}$ en saison sèche (Mars, Avril) et descendent à $10^{\circ} \mathrm{C}$ en saison froide (Décembre à Février). (Gomma, M'bareck, et al., 2017). 


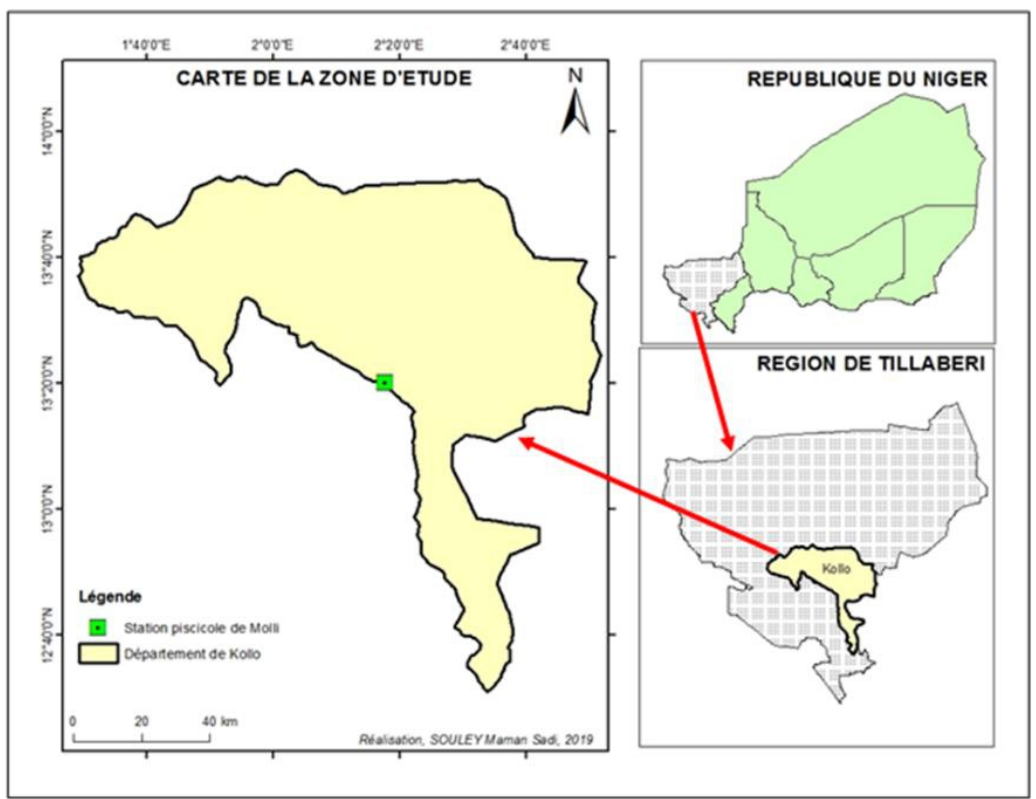

Figure 1: Carte du site d'étude

\section{Matériel végétal}

Le matériel végétal utilisé est la tomate (Lycopersicon esculentum Mill) de la variété Roma VF qui est une lignée très résistante au Verticillium et au Fusarium (VF). Son cycle de la plantation à la maturité est de 120 à 160 jours. Vigoureuse et productive, la plante produit des tomates allongées et ovales de couleur rouge de 60 à $70 \mathrm{~g}$ en moyenne qui peuvent atteindre 10 à $12 \mathrm{~cm}$. Leur chair ferme, douce et contenant peu d'eau est de bonne qualité.

\section{Dispositif expérimental}

L'essai a été conduit durant deux campagnes (2017 et 2018). Un dispositif expérimental en blocs complètement randomisés comprenant six (6) traitements y compris le témoin absolu organisés en 4 blocs soit 24 parcelles élémentaires a été utilisé. Chaque parcelle élémentaire a une superficie de 4,5 $\mathrm{m} 2$ (1,5 m de large x $3 \mathrm{~m}$ de long) et est constituée de quatre lignes séparées les unes aux autres de $0,5 \mathrm{~m}$ avec 6 plants par ligne soit $0,5 \mathrm{~m}$ entre les plants. Les 6 niveaux de traitements appliqués sont consignés dans le tableau1. 
Tableau 1: Les niveaux de traitements utilisés pour cette étude

\begin{tabular}{|c|c|c|c|}
\hline \multirow[b]{2}{*}{ Symboles } & \multirow[b]{2}{*}{ Traitements } & \multicolumn{2}{|c|}{ Dosage (kg) } \\
\hline & & $1 \mathrm{~m}^{2}$ & $\begin{array}{l}\text { Parcelle élémentaire } \\
\left(4,5 \mathrm{~m}^{2}\right)\end{array}$ \\
\hline T0 & $\begin{array}{l}0 \text { t. ha }{ }^{-1} \text { (contrôle } \\
\text { absolu) }\end{array}$ & 0 & 0 \\
\hline $\mathrm{T} 2$ & 20 t. ha- ${ }^{-1}$ BSC & 2 & 9 \\
\hline $\mathrm{T} 3$ & 30 t. ha- ${ }^{-1} \mathrm{BSC}$ & 3 & 13,5 \\
\hline $\mathrm{T} 4$ & 40 t. ha- ${ }^{-1}$ BSC & 4 & 18 \\
\hline $\mathrm{T} 5$ & 50 t. ha ${ }^{-1}$ BSC & 5 & 22,5 \\
\hline
\end{tabular}

FO : Fumure organique, BSC : Biomasse du Sida cordifolia

\section{Caractéristiques des matières organiques et du sol}

La biomasse du Sida cordifolia a été récoltée entre septembre et octobre de chaque campagne au stade de la fructification. Pour ce qui est de la fumure organique, elle a été ramassée dans le village de Molli près de la station où l'essai a été conduit. Cette fumure organique est constituée de déjections de bovins, des restants de la paille ayant servi de nourrir des bovins.

Avant l'incorporation de la biomasse du Sida cordifolia et de la fumure organique dans les différentes parcelles élémentaires, des échantillons du sol, de la BSC ainsi que de la FO ont été prélevés afin d'effectuer les analyses initiales.

Les caractéristiques physicochimiques initiales des matières organiques utilisées et du sol avant l'essai (2017) sont présentées dans le tableau 2.

Tableau 2 : Résultats des paramètres physico chimiques de matériaux utilisés ( \pm Écart type)

\begin{tabular}{|c|c|c|c|c|c|c|c|}
\hline & \multicolumn{7}{|c|}{ Éléments physico chimiques } \\
\hline & $\begin{array}{c}\text { pH-H2O } \\
(1: 2,5) \\
\end{array}$ & $\mathrm{C}(\%)$ & MO (\%) & $\begin{array}{c}\mathbf{N} \text { total } \\
(\%)\end{array}$ & $\begin{array}{c}\text { P total } \\
(\%)\end{array}$ & $\begin{array}{c}\text { K total } \\
(\%)\end{array}$ & $\mathbf{C} / \mathbf{N}$ \\
\hline \multicolumn{8}{|l|}{2017} \\
\hline Sol $(n=5)$ & $4 \pm 0,31$ & $0,75 \pm 0,24$ & $1,29 \pm 0,41$ & $0,04 \pm 0,02$ & $0,09 \pm 0,04$ & $0,12 \pm 0,076$ & $18,75 \pm 3,82$ \\
\hline $\begin{array}{l}\text { BSC } \\
(n=5)\end{array}$ & $4,81 \pm 0,10$ & $42,51 \pm 0,50$ & $73,24 \pm 0,30$ & $0,17 \pm 0,01$ & $0,13 \pm 0,01$ & $1,36 \pm 0,14$ & $250 \pm 17,2$ \\
\hline $\begin{array}{c}\text { FO } \\
(\mathbf{n}=\mathbf{5})\end{array}$ & $8,82 \pm 0,10$ & $10,92 \pm 0,20$ & $18,82 \pm 0,10$ & $0,17 \pm 0,004$ & $0,43 \pm 0,13$ & $1,52 \pm 0,23$ & $64,23 \pm 2,80$ \\
\hline \multicolumn{8}{|l|}{2018} \\
\hline $\begin{array}{c}\text { BSC } \\
(n=5)\end{array}$ & $5,1 \pm 0,10$ & $40,8 \pm 0,20$ & $59,2 \pm 0,40$ & $0,21 \pm 0,008$ & $0,17 \pm 0,01$ & $1,51 \pm 0,17$ & $194,28 \pm 8,30$ \\
\hline $\begin{array}{c}\text { FO } \\
(\mathbf{n}=5)\end{array}$ & $8,50 \pm 0,20$ & $10,14 \pm 0,30$ & $17,48 \pm 0,20$ & $0,25 \pm 0,02$ & $0,48 \pm 0,28$ & $1,33 \pm 0,10$ & $40,56 \pm 4,90$ \\
\hline
\end{tabular}

\section{BSC : Biomasse du Sida cordifolia ; FO : Fumure organique}

Ces résultats montrent que la biomasse du Sida cordifolia contient une quantité importante de la matière carbonée $(42,51 \%$ et $40,8 \%)$. Aussi, cette 
biomasse est acide avec un $\mathrm{pH}<6$ alors que la fumure organique utilisés est basique ( $\mathrm{pH}>8$ ). En 2017, la BSC et la FO contiennent plus de teneur en N, $\mathrm{P}$ et $\mathrm{K}$ que le sol (tableau 2). Pour ce qui est du sol, son $\mathrm{pH}$ est acide, il contient une teneur d'azote de $0,04 \%$ et un rapport $\mathrm{C} / \mathrm{N}$ de $18,75 \%$.

\section{Operations culturales}

La pépinière de la tomate a été installée à côté du site expérimental. Le paillage a été appliqué sur la pépinière (planches) pour éviter le dessèchement du sol et des graines. Après la levée, le paillage a été retiré et les planches ont été couvertes par une moustiquaire pour éviter les dégâts de criquets et de petits reptiles. En 2017, la pépinière a été installée le 17 octobre 2017 et en 2018, elle a été mise en place le 20 octobre 2018.

Le site expérimental a été labouré, disqué et nettoyé afin d'obtenir un sol profondément ameubli et aéré sur au moins $40 \mathrm{~cm}$ de profondeur et à structure homogène. Les blocs ont été délimités puis les différentes planches servant des parcelles élémentaires ont été confectionnées. La biomasse du Sida cordifolia L. a été enfouie dans le sol sur chacune des parcelles élémentaires sous forme de fumure de fond suivant le protocole expérimental deux semaines avant le repiquage soit 31 octobre 2017 et 03 novembre 2018 .

Chaque semaine, pour éviter la concurrence des mauvaises herbes, un désherbage manuel et à la binette est réalisé. L'irrigation se fait chaque trois jours jusqu'à un mois et après la fréquence est ramenée à une semaine. Pour protéger la culture les des maladies (champignon, virus), les des insectes et araignées, le TITAN (Hexaconazole) a été utilisé en raison de 1 litre/ha toutes les deux semaines.

\section{Observation et mesure des paramètres}

Du repiquage à la maturité, plusieurs paramètres regroupés en deux types ont été mesurés et ou observés : les paramètres du cycle de développement et ceux de production.

Pour les paramètres du cycle de développement, il s'agit du taux de survie de plants (TSP), la hauteur de plant à la floraison (HPf) en cm, la date de $50 \%$ floraison (DFl) en jour, le taux de floraison (TFl) en \%, le taux de fructification (Tfr) en \%, la date de la première récolte (DPr) en jour et la date de la dernière récolte (DDr) en jour.

Les dates ont permis de déterminer l'effet de l'application de la biomasse du Sida cordifolia sur la précocité et la durée de production de la cette variété de tomate. La date de la floraison a été relevée en fonction du nombre de jours, qui s'est écoulé du repiquage au jour où, $50 \%$ des pieds repiqués ont fleuri.

La date de la 1ère récolte, représente également, le nombre de jours qui s'est écoulé du repiquage au jour où s'est fait la lère récolte des fruits 
matures et sains. La date de la dernière récolte correspond au nombre de jours entre le repiquage et la dernière récolte. Ces deux dates ont permis de déterminer la durée de la production en jour qui est définie par le nombre de jours qui s'écoule entre la DPr et DDr.

Pour les taux de floraison et de la fructification, ils ont été estimés selon le code suivant: $0=$ absence de données : les plantes ne portent aucune fleurs ni de fruits ; $1=$ floraison très faible : nombre de fleur et de fruits inférieur à $5 ; 3=$ floraison faible : nombre de fleur et de fruits compris entre 5 et $25 ; 5=$ floraison moyenne : nombre de fleur et de fruits compris entre 25 et $50 ; 7=$ floraison forte : nombre de fleur et de fruits compris entre 50 et 75 et $9=$ floraison très importante : nombre de fleur et de fruits supérieurs à 75 (Kotaix, Angui, et al., 2013).

Quant aux paramètres de production, ils ont été évalués à travers : le Nombre moyen de fruits par pieds (NMf), il est déterminé sur les quatre pieds centraux de chaque parcelle. À chaque récolte, les fruits de ces pieds sont comptés ; le Poids moyen d'un fruit (PMf) en gramme (g). Cinq (5) fruits sont échantillonnés par hasard au niveau de chaque parcelle pour la pesée afin de déterminer le poids moyen d'un fruit ; le Rendement moyen en fruits (RdtMf) en tonne par hectare (t. ha-1).

\section{Échantillonnage et analyse du sol}

Après les deux campagnes successives (2017 et 2018), le sol de chaque traitement a été échantillonné et analysé afin de déterminer l'effet induit par les traitements sur les éléments physicochimiques $(\mathrm{N}, \mathrm{P}, \mathrm{K}, \mathrm{C}, \mathrm{MO}$, pH et le $\mathrm{C} / \mathrm{N})$ du sol.

\section{Analyse statistique}

Les valeurs moyennes pour chaque variable ont été testées pour leur distribution normale en utilisant le test de Shapiro-Wilk. Une analyse de la variance (ANOVA) au seuil de $5 \%$ a été effectuée pour tester si toutes les moyennes de traitements sont égales (hypothèse H0) ou s'il existe une différence significative entre les moyennes (hypothèse $\mathrm{H} 1$ : au moins une des moyennes est différentes des autres). Lorsqu'une différence significative est révélée, l'ANOVA est complétée par le test de Tukey.

Toutes ces analyses ont été faites à l'aide du logiciel MINITAB version 14.1. Certains graphiques ont été élaborés avec EXCEL 2016.

Analyse économique des traitements

Pour analyser la rentabilité économique des traitements, le rapport valeur sur coût (RVC) a été calculé. Ce rapport permet d'identifier le meilleur traitement facilement adoptable par les producteurs. Il est calculé à travers la formule suivante :

$\mathrm{RVC}=\frac{\text { valeur de l'augmentation du rendement par rapport au témoin en espèce }}{\text { Coût des fertilisants }}$ 
La collecte, le transport et l'incorporation de la biomasse du Sida cordifolia L., ont couté coûté 52212 FCFA / hectare ha-1. Pour le fumier, une somme de 50000 FCFA à l'hectare été dépensée. La main d'œuvre a été évaluée en homme/jour et a couté 57564 FCFA pour un hectare. Au moment de la récolte, le prix moyen d'un kilogramme de la tomate sur le marché était de 300 FCFA en 2017 et 350 FCFA en 2018.

\section{Résultats et discussions}

\section{Composition physicochimique du sol avant et après l'essai}

Les résultats de l'analyse initiale et finale $\mathrm{du}$ sol du site d'expérimentation sont présentés dans le tableau 3. D'après ces résultats, le sol est acide $(\mathrm{pH}=4)$ et contient une proportion de $0,04 \%$ d'azote. Pour ce qui est du rapport $\mathrm{C} / \mathrm{N}$, il est de l'ordre de 18,3 . Les teneurs $0,092 \%$ et $0,123 \%$ ont été obtenues respectivement pour le phosphore total et le potassium.

Une analyse de variance au seuil de 5\% a été réalisée sur les données des éléments physico chimiques du sol de chaque traitement après deux campagnes (tableau 3). Selon cette analyse, une différence significative entre les traitements a été observée pour l'ensemble des éléments analysés à l'exception le du $\mathrm{pH}(\mathrm{P}=0,132)$ dont les valeurs sont comprises entre 4,78 et 5,44 . Cela montre que les parcelles sont acides. Les parcelles les plus acide sont celles qui n'ont reçu aucun apport (T0=0 t/ha).

Tableau 1: Caractéristiques physicochimiques du sol avant et après l'essai en 2017

\begin{tabular}{|c|c|c|c|c|c|c|c|}
\hline \multirow[b]{2}{*}{ Modalités } & \multicolumn{7}{|c|}{ Éléments physico chimiques } \\
\hline & $\begin{array}{l}\text { pH-H2O } \\
(1: 2,5)\end{array}$ & $\mathrm{C}(\%)$ & MO (\%) & $\begin{array}{l}\text { N total } \\
(\%)\end{array}$ & $\begin{array}{l}\text { P total } \\
(\%)\end{array}$ & $\mathrm{K}$ total $(\%)$ & $\mathbf{C} / \mathbf{N}$ \\
\hline $\begin{array}{l}\text { Avant } \\
\text { l'essai }\end{array}$ & $4 \pm 0,31$ & $0,75 \pm 0,24$ & $1,29 \pm 0,41$ & $0,04 \pm 0,02$ & $0,092+0,04$ & $0,123+0,076$ & $18,30 \pm 3,82$ \\
\hline \multicolumn{8}{|c|}{ Après l'essai } \\
\hline T0 & $4,78^{\mathrm{a}}$ & $0,8^{\mathrm{a}}$ & $1,38^{\mathrm{a}}$ & $0,05^{\mathrm{a}}$ & $0,065^{\mathrm{a}}$ & $0,007^{\mathrm{a}}$ & $19,48^{\mathrm{c}}$ \\
\hline $\mathrm{T} 1$ & $5,03^{\mathrm{a}}$ & $0,75^{\mathrm{a}}$ & $1,29^{\mathrm{a}}$ & $0,07^{\mathrm{b}}$ & $0,122^{\mathrm{a}}$ & $0,037^{\mathrm{a}}$ & $11,54^{\mathrm{ab}}$ \\
\hline $\mathrm{T} 2$ & $4,97^{\mathrm{a}}$ & $0,87^{\mathrm{a}}$ & $1,5^{\mathrm{a}}$ & $0,07^{\mathrm{b}}$ & $0,079^{\mathrm{a}}$ & $0,134^{b}$ & $16,23^{b c}$ \\
\hline $\mathrm{T} 3$ & $5,25^{\mathrm{a}}$ & $1,17^{b c}$ & $2,01 \mathrm{bc}$ & 0,09 bc & $0,223^{a b}$ & $0,177^{b}$ & $13,35 \mathrm{abc}$ \\
\hline $\mathrm{T} 4$ & $5,09^{\mathrm{a}}$ & $1,31^{\mathrm{c}}$ & $2,25^{\mathrm{c}}$ & $0,10^{\mathrm{cd}}$ & 0,289 ab & $0,190^{b}$ & $12,71^{a b c}$ \\
\hline T5 & $5,44^{\mathrm{a}}$ & $0,94^{\mathrm{ab}}$ & $1,61^{\mathrm{ab}}$ & $0,12^{\mathrm{d}}$ & $0,457^{\mathrm{b}}$ & $0,198^{b}$ & $8,36^{\mathrm{a}}$ \\
\hline F pr. & $\mathbf{0 , 1 3 2}$ & $<.001$ & $<.001$ & $<.001$ & 0.020 & $<.001$ & 0,041 \\
\hline $\mathbf{C V}$ & 6,3 & 15,9 & 15,9 & 17,5 & 74,9 & 36,2 & 32,2 \\
\hline
\end{tabular}

T0 : Contrôle absolu (0 t/ha); T1 : 20 t. ha-1de la fumure organique ; T2 : 20 t. ha-1de la biomasse du Sida cordifolia L. ; T3 : 30 t. ha-1de la biomasse du Sida cordifolia L. ; T4 : 40 t. ha-1de la biomasse du Sida cordifolia L. ; T5 : 50 t. ha-1de la biomasse du Sida cordifolia

L. F pr: Probabilité de F

Verticalement, les moyennes suivies de mêmes lettres ne sont pas statistiquement différentes. 
La moyenne de carbone varie d'une parcelle à une autre $(\mathrm{P}<.001)$. La valeur maximale est obtenue au niveau de la parcelle ayant reçu le traitement $\mathrm{T} 4=40 \mathrm{t}$. ha-1 $\mathrm{BSC}(1,31 \%)$ et la minimale avec la parcelle $\mathrm{T} 1=20 \mathrm{t}$. ha-1 de la fumure organique $(0,75 \%)$ qui n'est pas statistiquement différente de la teneur de témoin absolu. La même tendance a été observée au niveau de la matière organique. Le taux de la matière organique est plus élevé dans la parcelle ayant reçu le traitement T4 (2,25\%). Le traitement T0, T1 et T2 contiennent des taux de matière organique statistiquement identiques. Pour ce qui est de l'azote, une différence hautement significative a été observée entre les parcelles $(\mathrm{P}=<.001)$. Le traitement $\mathrm{T} 5=50 \mathrm{t}$. ha- $1 \mathrm{BSC}$ contient plus de l'azote que tous les autres traitements. Le témoin absolu a enregistré la teneur la plus faible $(0,05 \%)$. Pour le phosphore total, la teneur varie de $0,065 \%$ (T0) à $0,457 \%$ (T5).

Les traitements $\mathrm{T} 0$ et $\mathrm{T} 1$ ont donnée des teneurs en potassium statistiquement identiques et les traitements T2, T3, T4 et T5 ont eu le même effet sur la teneur du sol en potassium. Le rapport $\mathrm{C} / \mathrm{N}$ le plus bas a été obtenu au niveau des parcelles ayant reçu le traitement T5.

\section{Effets de traitements sur le taux de survie de plants}

Le taux de survie de plants de la tomate diffère significativement entre les traitements $(\mathrm{F} . \mathrm{pr}<.001)$. Mais aucune différence significative n'a été révélée entre les années $(\mathrm{F} . \mathrm{pr}=0,59)$ et la combinaison traitement*années. (F.pr=0,58). Courant la campagne 2017, ce taux varie de 81,25\% (T0) à $96,87 \%$ pour le T4. En 2018, 98,96\% des plants du traitement T5 ont survécu contre $72,9 \%$ du témoin (Tableau 4) .

Tableau 4 : Résultats de l'ANOVA du taux de survie des plants selon les traitements et les campagnes.

\begin{tabular}{ccc}
\hline \multirow{2}{*}{ Traitements } & \multicolumn{2}{c}{ Taux de survie (\%) } \\
\cline { 2 - 3 } & $\mathbf{2 0 1 7}$ & $\mathbf{2 0 1 8}$ \\
\cline { 2 - 3 } T0 & $81,25^{\mathrm{ab}}$ & $72,92^{\mathrm{a}}$ \\
T1 & $87,5^{\mathrm{ab}}$ & $91,66^{\mathrm{ab}}$ \\
T2 & $89,58^{\mathrm{ab}}$ & $94,79^{\mathrm{b}}$ \\
T3 & $92,71^{\mathrm{b}}$ & $94,79^{\mathrm{b}}$ \\
T4 & $96,87^{\mathrm{b}}$ & $97,91^{\mathrm{b}}$ \\
T5 & $95,83^{\mathrm{b}}$ & $98,96^{\mathrm{b}}$ \\
\hline
\end{tabular}

F.pr

Années

0.598

Traitements

$<.001$

Années x Traitements

$\mathbf{0 . 5 8 0}$

T0 : Contrôle absolu (0 t/ha) ; T1 : 20 t. ha-1de la fumure organique ; T2 : 20 t. ha-1de la biomasse du Sida cordifolia L. ; T3 : 30 t. ha-1de la biomasse du Sida cordifolia L. ; T4 : 40 t. ha-1de la biomasse du Sida cordifolia L. ; T5 : 50 t. ha-1de la biomasse du Sida cordifolia

L. F pr: Probabilité de F

Verticalement, les moyennes suivies de mêmes lettres ne sont pas statistiquement différentes. 


\section{Effet de traitements sur la hauteur de plant de la tomate}

La hauteur des plants a été déterminée à la date de $50 \%$ floraison des parcelles.

Une différence hautement significative de hauteur (figure 2) a été obtenue entre les traitements (F.pr <.001) et entre les années (F.pr =0,001). Il ressort de l'analyse de la variance au seuil de 5\% que les hauteurs de plants à la floraison n'ont pas été influencées par la combinaison entre les traitements et les années (F.pr $=0,280$ ).

La hauteur du traitement témoin de 2017 est statistiquement identique à celle obtenue en 2018.

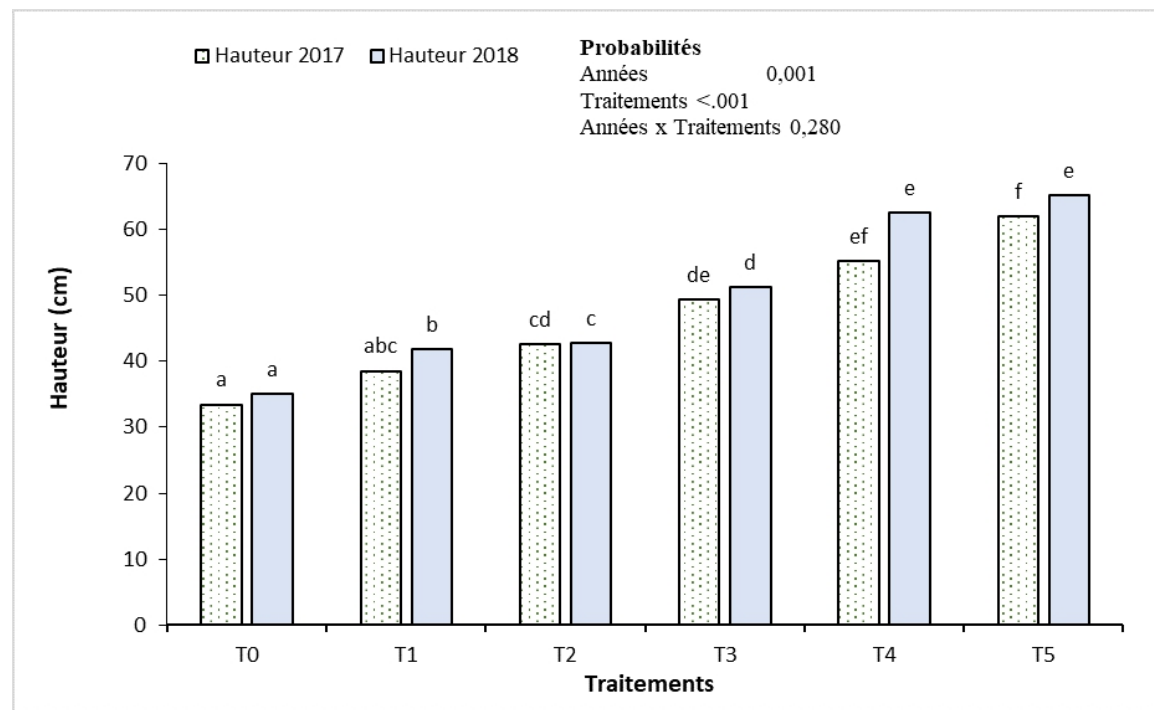

Figure 3 : Effet des traitements sur la hauteur de plants en 2017 et 2018

T0 : Contrôle absolu (0 t/ha); T1 : 20 t. ha-1de la fumure organique ; T2 : 20 t. ha-1de la biomasse du Sida cordifolia L. ; T3 : 30 t. ha-1de la biomasse du Sida cordifolia L. ; T4 : 40 t. ha-1de la biomasse du Sida cordifolia L. ; T5 : 50 t. ha-1de la biomasse du Sida cordifolia L. F pr: Probabilité de F

\section{Effet de traitements sur la floraison et la fructification}

L'analyse de variance au seuil de 5\% montre que les campagnes (2017 et 2018) et les traitements ont eu un effet significatif sur la date de $50 \%$ floraison $(\mathrm{P}=0,005)$, sur le taux de floraison $(\mathrm{P}=0,007)$ et sur celui de fructification $(\mathrm{P}=0,013)$.

D'une manière générale, une augmentation du taux de floraison a été obtenue selon la quantité de la biomasse du Sida cordifolia incorporée dans le sol.

Pour ce qui est du taux de fructification, en 2017 les traitements témoin absolu (T0) et le T1 (20t.ha-1 de la fumure organique) ont eu de taux \pm faible $(3,5)$. Le traitement $(\mathrm{T} 3)$ a enregistré un fort taux de fructification $(7,5)$. 
Le taux de fructification des plants en 2018 varie de \pm faible (code 3 ) à \pm fort (code 7). Les traitements T0 et T2 ont enregistré de taux de fructification \pm faible (code 3 ) et les parcelles ayant obtenu les traitements T3, T4 et T5 ont un taux de fructification \pm fort (code 7 ).

Tableau 5 : Effets de traitements sur la floraison et la fructification en 2017 et 2018.

\begin{tabular}{|c|c|c|c|c|c|c|}
\hline \multirow[t]{2}{*}{ Traitements } & \multicolumn{2}{|c|}{$\begin{array}{l}\text { Date de } 50 \% \\
\text { floraison }\end{array}$} & \multicolumn{2}{|c|}{$\begin{array}{c}\text { Taux de } \\
\text { floraison }\end{array}$} & \multicolumn{2}{|c|}{$\begin{array}{c}\text { Taux de } \\
\text { fructification }\end{array}$} \\
\hline & 2017 & 2018 & 2017 & 2018 & 2017 & 2018 \\
\hline T0 & $33,25^{b}$ & $32^{a b}$ & $3,8^{\mathrm{a}}$ & $5^{a b c}$ & $3,5^{\mathrm{a}}$ & $3,5^{\mathrm{a}}$ \\
\hline $\mathrm{T} 1$ & $33^{\mathrm{ab}}$ & $30^{\mathrm{ab}}$ & $4,5^{\mathrm{ab}}$ & $5,5^{\mathrm{abc}}$ & $3,5^{\mathrm{a}}$ & $3,5^{\mathrm{a}}$ \\
\hline $\mathrm{T} 2$ & $31,5^{\mathrm{ab}}$ & $28,5^{\mathrm{ab}}$ & $5^{a b c}$ & $6,5^{a b c}$ & $6^{\mathrm{ab}}$ & $7,5^{b}$ \\
\hline $\mathrm{T} 3$ & $29,75^{a b}$ & $26,75^{a b}$ & $6^{\mathrm{abc}}$ & $7 \mathrm{abc}$ & $7,5^{\mathrm{b}}$ & $8^{b}$ \\
\hline $\mathrm{T} 4$ & $29,25^{a b}$ & $26,25^{\mathrm{ab}}$ & $7^{a b c}$ & $8^{\mathrm{bc}}$ & $6^{\mathrm{ab}}$ & $8^{b}$ \\
\hline T5 & $28,5^{\mathrm{ab}}$ & $25,5^{\mathrm{a}}$ & $7^{a b c}$ & $8,5^{\mathrm{c}}$ & $5,5^{\mathrm{ab}}$ & $8^{b}$ \\
\hline \multicolumn{7}{|l|}{ F.pr } \\
\hline Années & \multicolumn{2}{|c|}{0,005} & \multicolumn{2}{|c|}{0.007} & \multicolumn{2}{|c|}{0.013} \\
\hline Traitements & \multicolumn{2}{|c|}{0,004} & \multicolumn{2}{|c|}{$<.001$} & \multicolumn{2}{|c|}{$<.001$} \\
\hline $\begin{array}{c}\text { Années x } \\
\text { Traitements }\end{array}$ & \multicolumn{2}{|c|}{0,99} & \multicolumn{2}{|c|}{0.997} & \multicolumn{2}{|c|}{0.376} \\
\hline
\end{tabular}

T0 : Contrôle absolu (0 t/ha) ; T1 : 20 t. ha-1de la fumure organique; T2 : 20 t. ha-1de la biomasse du Sida cordifolia L. ; T3 : 30 t. ha-1de la biomasse du Sida cordifolia L. ; T4 : 40 t. ha-1de la biomasse du Sida cordifolia L. ; T5 : 50 t. ha-1de la biomasse du Sida cordifolia L. F pr: Probabilité de F

Verticalement, les moyennes suivies de mêmes lettres ne sont pas statistiquement différentes.

\section{Effet de traitements sur les dates de récolte}

Les résultats du tableau 6, montrent que les années (2017 et 2018) et les traitements ont eu des effets hautement significatifs $(\mathrm{p}<.001)$ sur les dates de récoltes (première récolte et dernière de récolte).

Le date de la première récolte varie en 2017 de 53 JAR à 71 JAR et de 50 JAR à 66 JAR durant la campagne 2018. D'une façon globale, la date de la récolte de la campagne 2018 est plus précoce que celle de 2017.

En ce qui concerne la durée de récolte, en 2017, la récolte a duré de 99 JAR (T1) à 121 JAR (T3) et en 2018 de 106 JAR (T1) à 109 JAR (T2).

Tableau 6 : Effets de traitements sur les dates de récolte en 2017 et 2018

\begin{tabular}{clccc}
\hline \multirow{2}{*}{ Traitements } & \multicolumn{2}{c}{ Date de la récolte (JAR) } & \multicolumn{2}{c}{ Date de fin de récolte (JAR) } \\
\cline { 2 - 5 } & $\mathbf{2 0 1 7}$ & $\mathbf{2 0 1 8}$ & $\mathbf{2 0 1 7}$ & $\mathbf{2 0 1 8}$ \\
\hline T0 & $71,50^{\mathrm{e}}$ & $66,25^{\mathrm{de}}$ & $106,5^{\mathrm{abc}}$ & $108,2^{\mathrm{abcde}}$ \\
$\mathrm{T} 1$ & $64,50^{\mathrm{cde}}$ & $57,00^{\mathrm{abc}}$ & $99,5^{\mathrm{a}}$ & $106^{\mathrm{ab}}$ \\
$\mathrm{T} 2$ & $59,25^{\mathrm{bcd}}$ & $53,25^{\mathrm{ab}}$ & $115,2^{\mathrm{cdef}}$ & $109,2^{\mathrm{bcde}}$ \\
T3 & $58,00^{\mathrm{abcd}}$ & $52,75^{\mathrm{ab}}$ & $121^{\mathrm{f}}$ & $108,8^{\mathrm{bcde}}$ \\
T4 & $54,00^{\mathrm{ab}}$ & $50,00^{\mathrm{a}}$ & $117^{\mathrm{ef}}$ & $106^{\mathrm{ab}}$ \\
T5 & $53,00^{\mathrm{ab}}$ & $51,75^{\mathrm{ab}}$ & $116^{\text {def }}$ & $107,8^{\mathrm{abcd}}$ \\
\hline
\end{tabular}




\begin{tabular}{ccc}
\hline F,pr & & \\
\hline Années & $<.001$ & $<.001$ \\
Traitements & $<.001$ & $<.001$ \\
Années x & 0,629 & $<.001$ \\
Traitements & $0,0.9$
\end{tabular}

T0 : Contrôle absolu (0 t/ha); T1 : 20 t. ha-1de la fumure organique; T2: 20 t. ha-1de la biomasse du Sida cordifolia L. ; T3 : 30 t. ha-1de la biomasse du Sida cordifolia L. ; T4 : 40 t. ha-1de la biomasse du Sida cordifolia L. ; T5 : 50 t. ha-1de la biomasse du Sida cordifolia L. F pr: Probabilité de F

Verticalement, les moyennes suivies de mêmes lettres ne sont pas statistiquement différentes.

\section{Effets de traitements sur les paramètres de production}

Des différences significatives entre les traitements et entre les années ont été observées au niveau du nombre de fruit par pied (NMf) et du poids moyen d'un fruit (PMf).

En 2017, le témoin absolu a enregistré la plus petite valeur du nombre de fruit par pied (6 Fruits) et la plus grande valeur a été obtenue au niveau des parcelles ayant reçu le traitement T4 (24 fruits). La même tendance a été observée pour le poids moyen d'un fruit avec $46,56 \mathrm{~g}$ pour le témoin et 69,45 g pour le T4.

$\mathrm{Au}$ cours de la campagne 2018, le nombre de fruit par pied est important par rapport aux résultats de 2017. La plus petite valeur a été de 15 fruits par pieds (T0) et 35 fruits (T3) par pied a été la plus grande valeur. Pour le poids moyen d'un fruit, sa valeur varie de 56,5 g (T0) à 75,9 g (T5).

Tableau 7 : Effets de traitements sur les paramètres de production en 2017 et 2018.

\begin{tabular}{|c|c|c|c|c|}
\hline \multirow{2}{*}{ Traitements } & \multicolumn{2}{|c|}{ Nombre moyen de fruit par pied } & \multicolumn{2}{|c|}{ Poids moyen d'un fruit (g) } \\
\hline & 2017 & 2018 & 2017 & 2018 \\
\hline T0 & $6,36^{\mathrm{a}}$ & $15,05^{\mathrm{a}}$ & $46,56^{\mathrm{a}}$ & $56,5^{a b}$ \\
\hline $\mathrm{T} 1$ & $17,4^{\mathrm{b}}$ & $26,77^{\mathrm{b}}$ & $60,04^{a b}$ & $65,59^{a b}$ \\
\hline $\mathrm{T} 2$ & $16,83^{\mathrm{b}}$ & $22,68^{b}$ & $63,32^{a b}$ & $67,41^{a b}$ \\
\hline $\mathrm{T} 3$ & $17,56^{\mathrm{b}}$ & $35,5^{\mathrm{c}}$ & $63,32^{a b}$ & $70,03^{a b}$ \\
\hline $\mathrm{T} 4$ & $23,41^{\mathrm{c}}$ & $32,45^{\mathrm{c}}$ & $69,45^{a b}$ & $73,55^{b}$ \\
\hline T5 & $18,79^{b}$ & $27,19^{b}$ & $67,46^{\mathrm{ab}}$ & $75,9^{\mathrm{b}}$ \\
\hline \multicolumn{5}{|l|}{ F.pr } \\
\hline Années & \multicolumn{2}{|c|}{$<.001$} & \multicolumn{2}{|c|}{0,002} \\
\hline Traitements & \multicolumn{2}{|c|}{$<.001$} & \multicolumn{2}{|c|}{0,026} \\
\hline $\begin{array}{c}\text { Années x } \\
\text { Traitements }\end{array}$ & \multicolumn{2}{|c|}{0.341} & \multicolumn{2}{|c|}{0,986} \\
\hline
\end{tabular}

T0 : Contrôle absolu (0 t/ha); T1 : 20 t. ha-1de la fumure organique ; T2 : 20 t. ha-1de la biomasse du Sida cordifolia L. ; T3 : 30 t. ha-1de la biomasse du Sida cordifolia L. ; T4 : 40 t. ha-1de la biomasse du Sida cordifolia L. ; T5 : 50 t. ha-1de la biomasse du Sida cordifolia L. F pr: Probabilité de F

Verticalement, les moyennes suivies de mêmes lettres ne sont pas statistiquement différentes. 


\section{Rendement moyen en fruits}

Les rendements en fruits consommables enregistrés en 2017 et 2018 ainsi que le pourcentage de l'augmentation du rendement des traitements par rapport au rendement du témoin sont présentés dans le tableau 8.

Le rendement varie entre 10,02 t. ha- 1 et 24,3 t. ha- 1 en 2017. Il était inférieur à celui de 2018, qui allait de 13,82 t. ha-1 à 33,68 t. ha-1. Il ressort de l'analyse de variance que les traitements et les campagnes ont induit des variabilités importantes sur le rendement moyen de fruits consommables $(\mathrm{P}=<.001)$.

Tableau 8 : Effets de traitements sur le rendement moyen en fruits en 2017 et 2018.

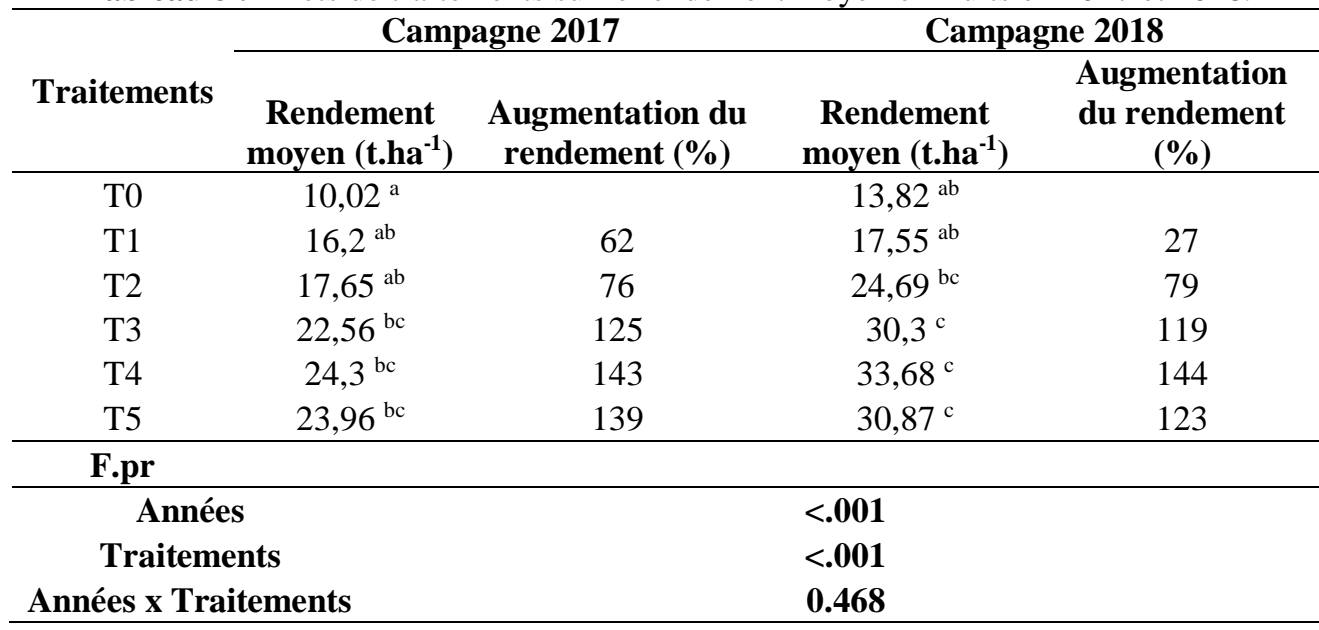

T0 : Contrôle absolu (0 t/ha); T1 : 20 t. ha-1de la fumure organique; T2 : 20 t. ha-1de la biomasse du Sida cordifolia L. ; T3 : 30 t. ha-1de la biomasse du Sida cordifolia L. ; T4 : 40 t. ha-1de la biomasse du Sida cordifolia L. ; T5 : 50 t. ha-1de la biomasse du Sida cordifolia L. F pr: Probabilité de F

Verticalement, les moyennes suivies de mêmes lettres ne sont pas statistiquement différentes.

\section{Rentabilité économique des traitements}

Les résultats de l'analyse économique de la culture de la tomate, variété Roma VF utilisant la biomasse du Sida cordifolia L. comme fertilisant sont consignés dans le tableau 9.

Tableau 9 : Rentabilité économique de différents traitements en 2017 et 2018.

\begin{tabular}{|c|c|c|c|c|c|c|}
\hline Campagnes & Traitement & $\begin{array}{c}\text { Coût } \\
\text { fertilisant } \\
\text { (FCFA) }\end{array}$ & $\begin{array}{c}\text { Rendement } \\
\text { moyen }\left(\text { t.ha }^{-1}\right)\end{array}$ & $\begin{array}{c}\text { Augmentation } \\
\text { du rendement } \\
(\text { FCFA) }\end{array}$ & $\begin{array}{c}\text { Valeur de } \\
\text { l'augmentation } \\
\text { du rendement } \\
\text { (FCFA) }\end{array}$ & RVC \\
\hline \multirow{4}{*}{2017} & $\mathrm{~T} 1$ & 1057564 & $16,2^{a b}$ & 6,18 & 1854000 & $1,75^{\mathrm{ab}}$ \\
\hline & $\mathrm{T} 2$ & 1101804 & $17,65^{\mathrm{ab}}$ & 7,63 & 2289000 & $2,08^{a b c}$ \\
\hline & $\mathrm{T} 3$ & 1623924 & $22,56^{\mathrm{bc}}$ & 12,54 & 3762000 & $2,32^{a b c}$ \\
\hline & $\mathrm{T} 4$ & 2146044 & $24,3^{\mathrm{bc}}$ & 10,02 & 3006000 & $1,40^{a b c}$ \\
\hline
\end{tabular}




\begin{tabular}{|c|c|c|c|c|c|c|}
\hline & $\mathrm{T} 5$ & 2668164 & $23,96^{b c}$ & 13,94 & 4182000 & $1,57^{\mathrm{a}}$ \\
\hline \multirow{5}{*}{2018} & $\mathrm{~T} 1$ & 1057564 & $17,55^{\mathrm{ab}}$ & 3,73 & 1305500 & $1,23^{\mathrm{a}}$ \\
\hline & $\mathrm{T} 2$ & 1101804 & $24,69^{\mathrm{bc}}$ & 10,87 & 3804500 & $3,45^{\mathrm{c}}$ \\
\hline & $\mathrm{T} 3$ & 1623924 & $30,3^{\mathrm{c}}$ & 16,48 & 5768000 & $3,55^{\mathrm{c}}$ \\
\hline & $\mathrm{T} 4$ & 2146044 & $33,68^{c}$ & 19,86 & 6951000 & $3,24^{\mathrm{bc}}$ \\
\hline & T5 & 2668164 & $30,87^{c}$ & 17,05 & 5967500 & 2,24 abc \\
\hline \multicolumn{7}{|c|}{ Probabilité } \\
\hline \multicolumn{2}{|c|}{ Années } & \multicolumn{3}{|c|}{$<.001$} & & 0,018 \\
\hline \multicolumn{2}{|c|}{ Traitements } & \multicolumn{3}{|c|}{$<.001$} & & $\mathbf{0 , 0 3 6}$ \\
\hline \multicolumn{2}{|c|}{ Années x Traitements } & & 0.468 & & & 0,326 \\
\hline
\end{tabular}

T0 : Contrôle absolu ( $0 \mathrm{t} / \mathrm{ha}) ;$ T1 : 20 t. ha-1de la fumure organique ; T2 : $20 \mathrm{t}$. ha-1de la biomasse du Sida cordifolia L. ; T3 : 30 t. ha-1de la biomasse du Sida cordifolia L. ; T4 : 40 t. ha-1de la biomasse du Sida cordifolia L. ; T5 : 50 t. ha-1de la biomasse du Sida cordifolia L. F pr: Probabilité de F

Verticalement, les moyennes suivies de mêmes lettres ne sont pas statistiquement différentes.

L'analyse de variance au seuil de $5 \%$ a révélé des variabilités significatives de RVC entre les traitements et les années $(\mathrm{P}<0,05)$. Les RVC obtenus en 2017 varient de 1,57 (T5) à 2,32 (T3) alors qu'en 2018, des valeurs de RVC plus importantes (de 1,23 à 3,55) ont été relevées respectivement avec $\mathrm{T} 1$ et $\mathrm{T} 3$.

Les résultats de 2017 indiquent que les T2; T3 et T4 ont statistiquement les mêmes RVC. En 2018, aucune différence significative n'a été obtenue entre les $\mathrm{T} 2$ et $\mathrm{T} 3$.

\section{Relation entre le RVC et la dose de la BSC}

La figure 4 montre qu'en 2017, il existe une corrélation négative moins étroite entre les doses appliquées et la rentabilité de leur emploi $\left(\mathrm{R}^{2}=0,5413\right)$ mais en 2018, cette relation est plus étroite $\left(R^{2}=0,717\right)$. En effet, les fortes doses de la biomasse du Sida cordifolia bien qu'augmentant le rendement, réduisent cependant leur rentabilité. 


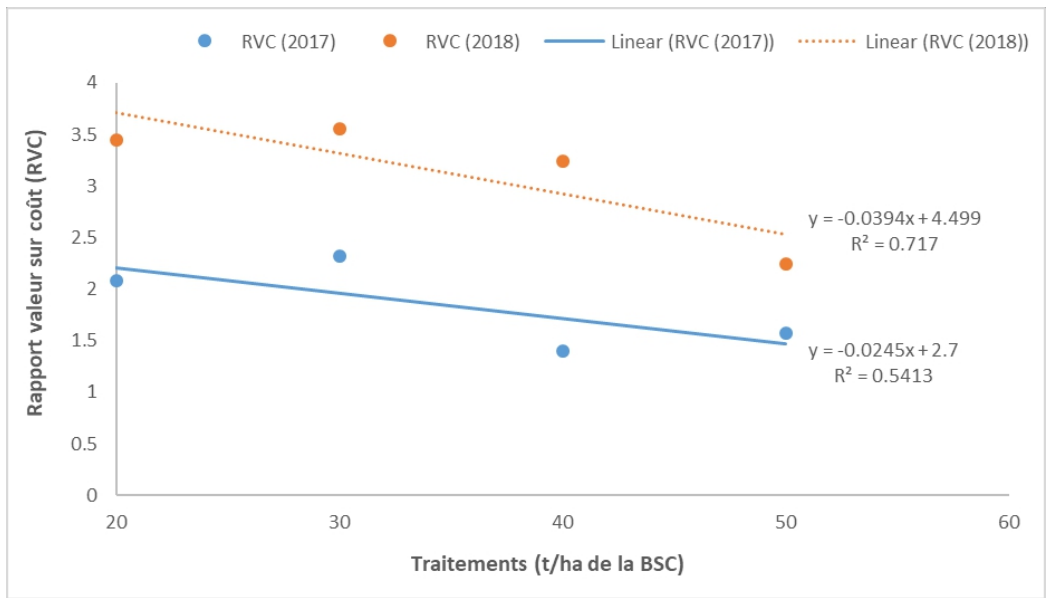

Figure 4 : Relation entre les doses de la BSC et leurs rentabilité économiques selon les campagnes (2017 et 2018).

\section{Discussions}

\section{Effet de l'incorporation de la biomasse du $S$. cordifolia $\mathbf{L}$. sur le sol}

D'après les résultats obtenus, le traitement T4 (40 t. ha-1 de la BSC) a donné après deux campagnes (2017 et 2018) le taux de carbone le plus élevé que les autres traitements. Le témoin absolu (T0), le T1 (20 t. ha-1 de la fumure organique), le T2 (20 t. ha-1 de la BSC) ont des taux de carbone statistiquement identique.

Quant à la teneur des sols en azote, elle est la plus élevée pour le traitement T5 $(0,12 \%)$ et la plus faible pour le témoin absolu $(0,05 \%)$. Au niveau des traitements T1, T2 aucune différence statistique n'a été révélée pour la teneur en azote.

Une augmentation des teneurs en phosphore au niveau des traitements T1, T3, T4 et T5 a été obtenue comparativement à la teneur initiale de cet élément. Cette tendance a été aussi observée pour ce qui est du potassium.

Ces résultats diffèrent de ceux de (Mugendi, Nair, et al., 1999, Shisanya, Mucheru, et al., 2009) qui ont montré que les taux d'azote, de phosphore et de potassium diminuent dans les sols amendés avec des engrais organiques et soumis à une culture continue. Toutefois, ces résultats corroborent les travaux de (F. Kaho, 2011) qui a obtenu des taux d'augmentation des paramètres physicochimiques du sol après l'incorporation des feuilles de Tithonia diversifolia pour la culture de maïs au Cameroun.

L'augmentation des taux des éléments observés entre le début et la fin de l'expérimentation pourrait être liée à la teneur initiale en carbone et en matière organique de la biomasse du Sida cordifolia qui ont des effets positifs sur les activités biologiques du sol. D'après (Robert, Antoine, et al., 2002), la matière organique, est le principal déterminant de l'activité biologique. La quantité, la diversité et l'activité de la faune et des micro-organismes sont en 
relation directe avec la présence de la matière organique. En effet, la matière organique et l'activité biologique qui en découle ont une influence majeure sur les propriétés physiques et chimiques des sols (Robert and Varet, 1996). L'agrégation et la stabilité de la structure du sol augmentent avec le contenu en carbone des sols. Le carbone des sols affecte aussi la dynamique et la biodisponibilité des principaux éléments nutritifs.

\section{Effet de traitements sur les paramètres de croissance et du développement de la tomate}

En 2017, la hauteur varie de 33,46 cm (T0) à 62,03 cm (T5) alors qu'en 2018, elle varie de 34,99 cm à 65,22 cm (figure 3). En plus de la teneur en azote du sol, le T5 (50 t. ha-1 de la BSC) a apporté une quantité de $85 \mathrm{~kg}$. ha1 de $\mathrm{N}$ et $105 \mathrm{~kg}$. ha- 1 de $\mathrm{N}$ respectivement en 2017 et 2018 alors que les parcelles témoins n'ont bénéficiaire d'aucun apport en azote.

Ces résultats confirment ceux de (Stevenson, Stevenson, et al., 1999) qui ont prouvé que l'azote stimule le développement et l'activité racinaire, favorisant ainsi l'exportation des autres éléments minéraux et la croissance des plantes. Les effets positifs d'une meilleure assimilation des nutriments favorisant la croissance des plantes a été observée par plusieurs auteurs (Duplessis and Envirocom, 2002, Fagnano, Adamou, et al., 2011). Ces résultats sont aussi en adéquation avec ceux de (Mouria, Ouazzani-Touhami, et al., 2010) qui ont mis en évidence lors d'une étude sur le compost, l'effet positif de différentes doses de composts de déchets d'ordures ménagères sur la croissance de la tomate.

Ainsi (Yin, Hayes, et al., 2012) ont montré qu'il existe une relation linéaire entre les hauteurs des plantes et la disponibilité du sol en azote. Le potassium et le phosphore apportés par la biomasse du Sida cordifolia interviennent aussi dans les mécanismes de croissance. En effet, plusieurs travaux ont montré que le potassium intervient dans la stimulation de la croissance via l'élongation foliaire (Hopkins, 2003, Mollier, 1999). En (Hopkins, 2003) conséquence, une faible teneur en phosphore et en potassium pourrait ralentir la croissance par réduction de la surface foliaire conformément aux travaux de (Jordan-Meille and Pellerin, 2004).

La faible croissance du témoin observée par rapport aux traitements, peut être due aux conditions physico-chimiques du sol. Selon (Mukalay, Shutcha, et al., 2008), la faible croissance des plantes peut être attribuée aux facteurs caractéristiques du sol, notamment le $\mathrm{pH}$ et les déficiences en nutriments. Le pH des parcelles témoins était de 4,78. Cette valeur peut entraîner des phénomènes de toxicité à cause d'une importante accumulation de fer (Ammari, Lamhamedi, et al., 2006).

L'incorporation de 30 t. ha-1, 40 t. ha- 1 et 50 t. ha- 1 de la biomasse du Sida cordifolia L. a permis d'obtenir respectivement des dates de $50 \%$ 
floraison de 29,75 JAR, 29,25 JAR et 28,5 JAR en 2017. Ce paramètre s'est amélioré en 2018 où une précocité des parcelles ayant reçu les traitements T3, T4 et T5 a été observé avec respectivement 26,75 JAR, 26,25 JAR et 25,5 JAR comme dates de $50 \%$ floraison. Les parcelles de témoin absolu ont été les plus tardives avec 33,25 JAR en 2017 et 32 JAR en 2018.

En 2017, les traitements T3, T4 et T5 ont permis aux parcelles d'avoir un taux de floraison \pm fort (code 6 et 7) par rapport au témoin qui a enregistré un faible taux de floraison $(3,8)$. La même tendance a été observé en 2018 où le taux de floraison varie de moyen (code 5) avec T0 au très important (code 9) au niveau de T5 (Tableau 6).

Les résultats montrent des taux de floraison et fructification plus élevés dans les parcelles amendées avec la BSC (nombre de fleurs et fruits supérieur à 75) que dans celles du témoin (nombre de fleurs et fruits compris entre 25 et 50). La précocité de la phase florale ainsi que la fructification élevée obtenues en 2018 pourraient être due à l'augmentation des éléments fertilisants dans le sol suite à l'incorporation de la BSC durant les deux campagnes.

En effet, après les deux campagnes, une augmentation du phosphore a été observée dans les parcelles où la BSC a été incorporée (tableau 3). Les parcelles ayant reçu les traitements à base de la BSC contiennent une teneur en phosphore variant de $0,079 \%$ à $0,457 \%$ supérieures à la teneur du témoin qui est de $0,065 \%$. Cet élément nutritif stimule la précocité de la floraison (Schvartz, Decroux, et al., 2005). Ces résultats sont aussi conformes à ceux de (OlumuyiwaTogun, Akanbi, et al., 2004) qui montre que la formation des fruits donc des boutons floraux et fleurs a été plus précoce chez les plants amendés par rapport aux témoins, ce qui pourrait sans doute induire également une précocité de la récolte.

\section{Effets de traitements sur les paramètres de production}

En 2017, le NMF varie de 6 à 23 fruits par pieds. Ces valeurs ont été respectivement obtenues au niveau des plants ayant reçu les traitements $\mathrm{T} 0$ et T4. Pour ce qui est de la campagne 2018, un NMF plus important selon le traitement a été obtenu comparativement à l'année 2017. Les plants du témoin ont donné en moyen 15 fruits par pieds alors qu'au niveau du T3, il a été enregistré une moyenne de 35 fruits par pied. Ces résultats sont supérieurs à ceux obtenu par (Mouria, Ouazzani-Touhami, et al., 2010) pour les plantes de tomate cultivées sur différentes doses de composts d'ordures ménagères où ils ont eu le nombre de fruits variant de 1 à 3 .

(Zuang, 1984) montre que le nombre de fruit est lié directement au nombre de fleurs fonctionnelles et à la réussite de la fécondation. Pour cette étude, il a été obtenu des taux de floraison variables et élevés dans les parcelles amendées. Cela montre que les différences en nombre de fruits ne peut donc 
être liée qu'à l'importance du taux de la floraison observée suite à l'augmentation des teneurs en $\mathrm{N}, \mathrm{P}$ et $\mathrm{K}$ dans les parcelles amendées.

En 2018, le poids moyen d'un fruit de $69,45 \mathrm{~g}$ et $46,56 \mathrm{~g}$ ont été obtenus respectivement au niveau de T4 et T0. Ces valeurs sont plus élevées comparativement à celles de 2017 où elles varient de $6,36 \mathrm{~g}$ pour les fruits de T0 à 23, $41 \mathrm{~g}$ au niveau des fruits de T4. L'incorporation de 40t. ha-1de la BSC (T4) correspond à un apport de $4 \mathrm{~kg}$. ha-1 de $\mathrm{N}, 11,56 \mathrm{~kg}$. ha-1 de $\mathrm{P}$ et $7,6 \mathrm{~kg}$. ha-1 de K. Les effets obtenus sur les paramètres de production suite à l'incorporation de la biomasse du Sida cordifolia dans le sol peut être expliqué par l'augmentation et la disponibilité des éléments minéraux. En effet, (Cobo, Barrios, et al., 2002, Palm, 1995) ont montré que le taux de décomposition de la matière organique et l'augmentation des rendements étaient étroitement lié à la synchronisation entre la libération des nutriments et leur assimilation par la plante.

En 2017, le rendement le plus élevé a été obtenu au niveau du traitement T4 (24,3 t. ha-1) suivi du T5 (23,96 t. ha-1 ). Le traitement T1 a un rendement de 16,2 t. ha- 1 un peu moins du rendement de T2 qui était de 17,65 t. ha-1. Le témoin absolu (T0) a enregistré le plus faible rendement avec 10,02 t. ha-1. Pour la campagne 2018, le rendement varie de 13,82 t. ha-1 pour le témoin absolu (T0) à 33,68 t. ha-1. (T4).

En 2017, l'application de la fumure à 20t. ha-1 a augmenté le rendement de $62 \%$ alors que l'incorporation de 40 t. ha-1 de la biomasse du Sida cordifolia a induit une augmentation de $143 \%$ par rapport au témoin absolu. La même tendance a été observé sur les résultats de la campagne 2018 avec des pourcentages d'augmentation du rendement allant de $27 \%$ à $144 \%$ obtenus respectivement au niveau de traitement $\mathrm{T} 1$ et $\mathrm{T} 4$.

D’une manière générale, le rendement a augmenté dans les parcelles amendées avec la biomasse du Sida cordifolia par rapport aux témoins. Ces résultats corroborent l'étude de (Abbasi, Al-Dahmani, et al., 2002) qui ont montré que, en plus de la stimulation de la croissance des plants de tomate, une augmentation du rendement a été obtenue dans les systèmes de production organique.

La faible production des parcelles témoins peut être attribuée aux facteurs caractéristiques des sols acides $(\mathrm{pH}=4,78)$, déficiences en nutriments (Mulaji Kyela, 2011). En plus, sur les parcelles témoins, l'absence d'apports organiques s'accompagne d'une perte en matières organiques et en nutriments, d'une acidification des sols, d'une réduction de la biomasse et de l'activité microbienne, d'une insolubilisation du phosphore qui ensemble contribuent à la baisse sensible des rendements des cultures (Deblay, 2006). 


\section{Rentabilité économique de la culture de tomate amendée avec la BSC}

Selon la (FAO/IFA., 2000)., le RVC doit être au moins égal à 2 pour permettre aux producteurs de couvrir les frais directs liés à l'utilisation des fertilisants. Les résultats de la campagne 2017 montrent que seules l'application de 20 et 30 tonnes de la biomasse du Sida cordifolia sont économiquement rentables pour la culture de la tomate leurs RVC sont supérieurs à 2. Par contre, les traitements T1, T4 et T5 n'ont pas été rentable (tableau 9).

Pour ce qui est de la campagne 2018, la rentabilité économique a été obtenue au niveau de tous les traitements appliquant la biomasse du Sida cordifolia $(20 ; 30 ; 40$ et 50 t. ha-1). Seul, le traitement T1 (20 t. ha-1 de la fumure organique) a donné un RVC inférieur à 2 .

Les résultats de deux campagnes (2017 et 2018) montrent que le traitement T3 (30 t. ha-1 de la BSC) a toujours était plus rentable que les autres traitements. Bien qu'en 2018, toutes les parcelles ayant été amendées par la biomasse du Sida cordifolia avaient des RVC supérieurs à 2, cette étude montre que le traitement $\mathrm{T} 3$ est le plus recommandé aux producteurs.

La relation entre les doses de la biomasse du Sida cordifolia et la rentabilité économique a montré que plus la dose augmente, moins la rentabilité est importante. Ces résultats sont similaires à ceux de (Yannick, Minerve, et al., 2013) qui ont trouvé que le RVC de l'emploi des déchets humains recyclés diminuent avec la dose. Ceci serait lié au coût de production élevée qui aurait réduit le bénéfice net. Des résultats similaires, montrant que les apports de faibles doses de Tithonia diversifolia accroissent la rentabilité des cultures ont été obtenus (Chukwuka and Omotayo, 2009, Nziguheba, Merckx, et al., 2002).

\section{Financement}

Les auteurs remercient la Norvège d'avoir financé cette recherche à travers son ambassade basée à Bamako au Mali.

\section{Conclusion}

L'incorporation de la biomasse du Sida cordifolia à différentes doses dans le sol a augmenté de façon significative les caractéristiques physicochimiques du sol après deux campagnes successives de la tomate. Les différentes doses testées ont montré des performances agronomiques meilleures par rapport aux témoins absolu et au traitement T1 (20 t. ha-1 de la fumure organique). Des effets positifs des traitements ont été enregistrés sur les paramètres de croissance, du développement ainsi que sur ceux du rendement. Une évolution croissante des rendements en fonction de l'augmentation de la dose de la BSC a été observée. Des taux d'augmentation 
du rendement par rapport aux témoins absolu variant de $62 \%$ à $139 \%$ en 2017 et de $27 \%$ à $144 \%$ en 2018 ont été obtenus.

La détermination de la rentabilité économique de l'utilisation de la biomasse du Sida cordifolia comme fertilisant pour la culture de la tomate a révélé que la dose de 30 t. ha-1 est la plus rentable. Cela est capital pour les petits producteurs qui ont un pouvoir d'achat très bas.

Dans les conditions de cette étude, la biomasse du Sida cordifolia utilisée comme fertilisant pourrait constituer une alternative réelle à l'utilisation démesurée des engrais chimiques dans le système maraicher au Niger. La BSC présente un grand potentiel pour l'amélioration de la qualité du sol et peut fournir des éléments nutritifs nécessaires à la culture de la tomate sans apport d'engrais minéraux.

Cette étude est d'autant plus intéressante que l'adoption de la fertilisation à base de la biomasse du Sida cordifolia pourrait permettre à la fois d'augmenter durablement la production de la tomate et réduire de la prolifération du Sida cordifolia L. dans le terroir.

\section{References:}

1. Abbasi, P., J. Al-Dahmani, F. Sahin, H. Hoitink and S. Miller. 2002. Effect of compost amendments on disease severity and yield of tomato in conventional and organic production systems. Plant disease 86: 156161.

2. Adam, T. 1995. Etude de deux parasites d'origine tellurique sur le niebe : Macrophomina phaseolina Goïd et Striga gesneroïdes Willd. Thèse de Doctorat es sciences naturelles, Université Abdou Moumouni, 102p.

3. Adamou, H., M. Garba, M. Mairo, B. Adamou, S. Oumarou, A. Kimba, et al. 2017. Geographical distribution of the tomato borer, Tuta absoluta Meyrick (Lepidoptera: Gelechiidae) in Niger. Scholars Academic Journal of Biosciences 5: 108-113.

4. Ammari, Y., M.S. Lamhamedi, N. Akrimi and A.Z. El Abidine. 2006. Qualités physiologiques de jeunes plants de Pin d'Alep élevés en pépinière moderne sur différents substrats à base de compost. Geo Eco Trop 30: 11-24.

5. Chukwuka, K. and O. Omotayo. 2009. Soil fertility restoration potentials of Tithonia green manure and water hyacinth compost on a nutrient depleted soil in South Western Nigeria using Zea mays L. as test crop. Research journal of soil biology 1: 20-30.

6. Cobo, J., E. Barrios, D.C. Kass and R. Thomas. 2002. Nitrogen mineralization and crop uptake from surface-applied leaves of green manure species on a tropical volcanic-ash soil. Biology and fertility of soils 36: 87-92. 
7. Deblay, S. 2006. Fertilisation et amendements. Educagri éditions, 2ème édition, Paris, $129 \mathrm{p}$.

8. Djiéto-Lordon, C., D. Alene and J. Reboul. 2007. Contribution à la connaissance des insectes associés aux cultures maraîchères dans les environs de Yaoundé-Cameroun. Cameroon J Biol Biochem Sci 15: 113.

9. Duplessis, J. and N. Envirocom. 2002. Le compostage facilité: guide sur le compostage domestiqueMontréal: NOVA Envirocom. 107 p.

10. F. Kaho, M.Y., P. Feujio-Teguefouet J.C. Tchantchaouang. 2011. Effet combiné des feuilles de Tithonia diversifolia et des engrais inorganiques sur les rendements du maïs et les propriétés d'un sol ferralitique au Centre Cameroun. Tropicultura 29: 39-45.

11. Fagnano, M., P. Adamo, M. Zampella and N. Fiorentino. 2011. Environmental and agronomic impact of fertilization with composted organic fraction from municipal solid waste: a case study in the region of Naples, Italy. Agriculture, ecosystems \& environment 141: 100107.

12. FAO/IFA. 2000. Fertilizers and their use - A pocket guide for extension officers. . Fourth edition. ed. FAO and IFA. 70 p.

13. Gomma, A.D., M. M'bareck, S. Ayssiwede, I. Salissou, M. Mahamadou, S. Siddo, et al. 2017. Evaluation technique et economique des formules alimentaires de blocs multi nutritionnels d'embouche ovine au Niger. Agronomie Africaine 29: 1-11.

14. Hopkins, W. 2003. Physiologie Végétale. Edition de Boeck, Université de Bruxelles : Belgique, $532 \mathrm{p}$.

15. Jordan-Meille, L. and S. Pellerin. 2004. Leaf area establishment of a maize (Zea mays L.) field crop under potassium deficiency. Plant and Soil 265: 75-92.

16. Kiari, A.S., I. Aboubacar and B. Habsatou. 2013. Test de complément minéral foliaire sur le riz au niveau de l'aménagement hydro-agricole de Saga et sur les cultures maraichères sur les sites de Gamkalé et Yantala corniche. p. 18.

17. Kotaix, A., P. Angui, C. Pierre, N. Diby, D. Dao and B. Bonfoh. 2013. Effet de l'engrais organique liquide «Dragon 1», sur le developpement de la tomate au sud et au centre-ouest de la Côte D'Ivoire. Agronomie Africaine 25: 37-52.

18. Laterrot, H. 1994. Création de Populations Sources pour la Sélection de Variétés de Tomates Résistantes à la Virose de L'enroulement Foliaire dans les Pays Méditerranéens Subtropicaux et Tropicaux. INRA-Station d'amélioration des plantes maraîchères. INRA-Station d'amélioration des plantes maraîchères. MontFavet-France, BP: 9484143. 
19. Michel, H., G. Pierre, T. Sabi Bira Joseph, B. Julien and E. Michée Iboukoun. 2016. Effet des engrais organiques sur la croissance et le rendement de deux variétés de tomate (Lycopersicum esculentum) dans la commune de Parakou (Nord Bénin). International Journal of Innovation and Scientific Research 24 No: 86-94.

20. Mollier, A. 1999. Croissance racinaire du maïs (Zea mays L.) sous déficience en Phosphore. Etude expérimentale et modélisation. Thèse de doctorat. Université de Paris XI Orsay. Paris, France. 200 p.

21. Mouria, B., A. Ouazzani-Touhami and A. Douira. 2010. Valorisation agronomique du compost et de ses extraits sur la culture de la tomate. Rev. Ivoir. Sci. Technol 16: 165-190.

22. Mugendi, D., P. Nair, J. Mugwe, M. O'neill and P. Woomer. 1999. Alley cropping of maize with calliandra and leucaena in the subhumid highlands of Kenya: Part 2. Soil-fertility changes and maize yield. Agroforestry systems 46: 39-50.

23. Mukalay, M., M. Shutcha, K. Tshomba, K. Mulowayi, C. Kamb and L. Ngongo. 2008. Causes d'une forte hétérogénéité des plants dans un champ de maïs dans les conditions pédoclimatique de Lubumbashi. Presses universitaires de Lubumbashi. Annales Faculté des Sciences Agronomiques 1: 4-11.

24. Mulaji Kyela, C. 2011. Utilisation des composts de biodéchets ménagers pour l'amélioration de la fertilité des sols acides de la province de Kinshasa (République Démocratique du Congo). (Thèse de Doctorat). Gembloux, Belgique, Université de Liège, Gembloux Agro-Bio Tech., 172 p.

25. Nziguheba, G., R. Merckx and C. Palm. 2002. Combining Tithonia diversifolia and fertilizers for maize production in a phosphorus deficient soil in Kenya. Agroforestry systems 55: 165-174.

26. OlumuyiwaTogun, A., W.B. Akanbi and J.A. Adediran. 2004. Growth, nutrient uptake and yield of tomato in response to different plant residue composts. JOURNAL OF FOOD AGRICULTURE AND ENVIRONMENT 2: 310-316.

27. Palm, C.A. 1995. Contribution of agroforestry trees to nutrient requirements of intercropped plants. Agroforestry: Science, Policy and Practice. Springer. p. 105-124.

28. RECA. 2018. Résultats définitifs de l'enquête sur les productions horticoles 2017/2018. Ministere de l'Agriculture et de l'Elevage, https://reca-niger.org/spip.php?article1220. p. 58.

29. Robert, M., J. Antoine, F. Nachtergaele, J. Benites, R. Brinkman, R. Dudal, et al. 2002. La séquestration du carbone dans le sol pour une meilleure gestion des terres-Rapport sur les ressources en sols du 
monde (96)-FAO. URL (Déc. 2008): http://www. fao. org/docrep/005/y2779f/y2779f00. htm\# toc.

30. Robert, M. and J. Varet. 1996. Le Sol: interface dans l'environnement: ressource pour le développement, Editions Masson, Collection Sciences de l'environnement, $244 \mathrm{p}$.

31. Schvartz, C., J. Decroux and J.-C. Muller. 2005. Guide de la fertilisation raisonnée: grandes cultures et prairiesFrance Agricole Editions. 412 p.

32. Shisanya, C.A., M.W. Mucheru, D.N. Mugendi and J.B. Kung'u. 2009. Effect of organic and inorganic nutrient sources on soil mineral nitrogen and maize yields in central highlands of Kenya. Soil and Tillage Research 103: 239-246.

33. Stevenson, F.J., E. Stevenson and M. Cole. 1999. Cycles of soils: carbon, nitrogen, phosphorus, sulfur, micronutrientsJohn Wiley \& Sons. $448 \mathrm{p}$.

34. Weinberger, K. and T.A. Lumpkin. 2007. Diversification into horticulture and poverty reduction: a research agenda. World development 35: 1464-1480.

35. Yannick, U.S., C.K. Minerve, T.K. John, M.M. Emmanuel, K.K. Prisca, N.N. François, et al. 2013. Utilisation des déchets humains recyclés pour l'augmentation de la production du maïs (Zea mays L.) sur un ferralsol du sud-est de la RD Congo. Journal of Applied Biosciences 66: 5070-5081.

36. Yin, X., R.M. Hayes, M.A. McClure and H.J. Savoy. 2012. Assessment of plant biomass and nitrogen nutrition with plant height in early-to mid-season corn. Journal of the Science of Food and Agriculture 92: 2611-2617.

37. Zuang, H. 1984. Les aléas climatiques liés à la production quantitative et qualitative de la tomate. Dans: Agrométéorologie et productions légumières. Les Colloques de l'INRA 33: 77-79. 